\title{
Depressive episodes-evidence for a causal role of primary anxiety disorders?
}

\author{
Hans-Ulrich Wittchen ${ }^{\text {a,b, }}$, Katja Beesdo ${ }^{\text {a }}$, Antje Bittner ${ }^{\text {a }}$, Renee D. Goodwin ${ }^{c}$ \\ a Institute of Clinical Psychology and Psychotherapy, Dresden University of Technology, \\ Chemnitzerstrasse 46, 01187 Dresden, Germany \\ b Max Planck Institute of Psychiatry, Munich, Germany \\ c Department of Epidemiology, Columbia University, Mailman School of Public Health, New York, \\ NY, USA
}

\begin{abstract}
Anxiety and depressive disorders are common mental disorders in general population, imposing tremendous burden on both affected persons and society. Moreover, comorbidity between anxiety and depressive conditions is high, leading to substantial disability and functional impairment. Findings consistently suggest that anxiety disorders are primary to depression in the majority of comorbid cases. Yet, the question of whether anxiety disorders are risk factors for depression, and potentially even causal risk factors for the first onset of depression, remains unresolved. Recent results have shown that anxiety disorders increase the risk for subsequent depression, and also affect the course of depression, resulting in a poorer prognosis. Further, some results suggest a dose-response-relationship in revealing that a higher number of anxiety disorders and more severe impairment associated with anxiety disorders additionally increase the risk for subsequent depression. The goal of this paper is to review recent literature, summarize implications of previous findings, and suggest directions for future research regarding preventive and intervention strategies.
\end{abstract}

Keywords: Anxiety; Depression; Comorbidity; Risk factor

\section{Introduction}

Anxiety and depressive disorders have been found to be associated with extremely high health economic costs. Fairly comprehensive comparative analyses have revealed that among all mental disorders, anxiety disorders are associated with the highest total costs, followed by affective disorders (330.373 millions of US dollars) [37]. It is noteworthy that for affective disorders, the largest proportion of these costs are direct costs (e.g. related to treatment and intervention), whereas the bulk of costs of anxiety disorders occurs primarily in indirect costs (e.g. value of lost output resulting from reduced productivity, disability, etc.). This remarkable difference has been attributed to the higher prevalence, earlier age of onset, and more frequent chronic courses of anxiety disorders in combination with the lower proportion of patients with anxiety disorders receiving any treatment. The increasing evidence for such tremendous, yet differential, diagnosis-specific health economic impact and the fact that anxiety and depressive disorders frequently occur together $[20,24,46]$ has made the search for more effective preventive and treatment strategies a high priority issue in research. In the core of such considerations is the question of the degree to which anxiety disorders precede depressive disorders and whether it is possible to reduce not only the incidence of anxiety disorders and to improve their natural course by means of earlier and more aggressive treatment, but also whether it is possible to reduce the incidence of secondary depression. This important second goal refers to the attractive speculation that anxiety disorders as predominantly early manifestations of mental disorders might constitute a causal risk factor 
for the subsequent development of depressive disorders and a more malignant further course in terms of more frequent depressive episodes, longer duration of episodes, and a higher frequency of chronic depression.

A significant obstacle in this respect has been the lack of convincing data that support the assumption of the causal role of primary anxiety disorders as an important pathway toward the prevention of the onset of secondary depression. Further, there are few available data to date that provide clues for the core aetiopathogenic processes involved in this pattern.

This paper reviews the available evidence for these claims primarily using data from epidemiological surveys, focusing in particular on prospective-longitudinal findings in community samples.

\section{The epidemiology of anxiety and depressive disorders}

\subsection{Prevalence}

Data from representative community samples carried out across the world (USA, Canada, Switzerland, Netherlands, Norway, Germany, Italy, Great Britain, Australia) have revealed quite consistently that anxiety and depression are frequent mental disorders with lifetimeprevalences for any anxiety disorder ranging from $15 \%$ to $25 \%$ and for any depressive disorder ranging from $10 \%$ to $18 \%$ [1,3,11,12,16,23,29,33,38,41,42,58].Among the anxiety disorders, specific phobias are diagnosed most frequently, followed by social phobia. The prevalence estimates for generalized anxiety disorder (GAD) and agoraphobia are somewhat lower, but substantial (4-6\%). Obsessive-compulsive disorder and panic disorder are less frequent (1-3.5\%). Fewer studies have reported prevalence rates for posttraumatic stress disorder (PTSD) and most of these findings have shown considerably wider variation with estimates ranging between $1.3 \%$ and $1.6 \%$ in Germany [11,33,58], and $8.4 \%$ in the US [41]. In comparison with these lifetime rates for anxiety disorders, 12-month estimates are generally lower, yet the pattern of relative prevalence rates of the specific anxiety disorders is consistent across studies. Among depressive disorders, major depressive episodes (MDE) are most frequent (9-17\%), whereas the prevalence estimates of dysthymia range between $2 \%$ and $6 \%[3,23,33,41,42]$.

\subsection{Onset of anxiety and depressive disorders}

Also consistent across both cross-sectional retrospective and prospective incidence studies, anxiety disorders have consistently been found to have an early onset dated in childhood, adolescence or early adulthood [56]. Except for GAD, panic disorder, and agoraphobia, which may occur with differing incidence patterns, both in adolescence and more frequently in later life, the typical age at risk for developing a phobia is clearly childhood or early adolescenceonset after age of 20 years seems to be a rare phenomenon [30,44,49,56]. Among all anxiety disorders, the pattern of onset with GAD seems to be remarkably different from that seen with other anxiety disorders. In most studies, prevalence rates for GAD in adolescents and young adults are usually low and then increase substantially with age [51]. In fact, analysis of the National Comorbidity Survey (NCS, USA) and German National Health Interview and Examination Survey-Mental Health Supplement (GHS) seem to suggest that GAD appears to be an anxiety disorder with consistently later onset, relative to phobias for instance, and appears more similar to the onset characteristics seen in affective disorders [8,59]. Yet, it should be noted that this does not necessarily indicate that individuals with onset of GAD in adulthood have been healthy prior to meeting the full criteria. Wittchen et al. [54] 
demonstrated that the majority of all GAD cases had been suffering from at least one childhood or adolescent anxiety disorder of other type prior to GAD onset.

In remarkable contrast to anxiety disorders, major depressive disorders have their first onset considerably later in late adolescence, early adulthood or even late life. Findings from the International Consortium of Psychiatric Epidemiology (ICPE) Surveys revealed that the median age of onset of MDE is in the early to mid-twenties and thus about 5-8 years later than the mean age for anxiety disorders. Unlike anxiety disorders (except for GAD), the risk of MDE continues throughout the life course [1]. According to data from the epidemiologic catchment area (ECA) study, the highest hazard rates occurred for both females and males at the ages 15-19 and 25-29 years [7]. Similar findings have also been reported from prospective-longitudinal studies in young adults [34].

\subsection{Patterns of course}

Both anxiety and depressive disorders also reveal remarkable differences with regard to course.

The most frequent course of anxiety disorders can be described as chronic-mild and persistent [45]. This characterization, however, is frequently misunderstood as meaning that each type of anxiety disorder, if present, persists on the threshold level over years or even the lifecourse. This is not the case; in fact, the typical picture is a waxing and waning pattern of symptomatology (symptomatic, subthreshold, threshold) with frequent syndromal shifts within the anxiety spectrum (shifts from specific to social phobia, from panic/agoraphobia to GAD or other phobias, etc.) [55]. The course of social anxiety disorder, for example, has been recently examined in a 3-wave follow-up study of 3021 adolescents and young adults [58]. The majority of cases of social anxiety disorder had their onset before the age of 18 years [47], and not infrequently even earlier in childhood. Until that time, the disorder was in the vast majority described as already progressive worsening or persistent: However, there was a considerable degree of waxing and waning in symptomatology, most likely due to challenges in life. Stable remission was found to be rare and limited to less than $20 \%$. Similar findings are available from the ECA study covering almost the whole lifespan: rates of remission (defined as: free of symptoms during the past year) were $42 \%$ in panic disorder and $38 \%$ in phobias. The mean duration of panic disorder (in remitted cases) is 7.1 years. Phobias have a longer duration of 15.4 years and subjects with obsessive-compulsive disorder had this disorder for 6.4 years [10,38]. Regarding GAD, more than $40 \%$ of subjects had the disorder for more than 5 years and more than $10 \%$ for more than 20 years [5].

This relatively high degree of symptom stability among anxiety disorders is in sharp contrast with that typically found for depressive disorders in the community.

The predominant pattern among community cases with depression is rather episodic with an average length of 3 months per episode [39,57] and, at least in the first three decades of life, chronic depression constitutes only a small proportion of all persons affected. Overall, the best estimate seems to be that one-third remit completely with no further residual symptoms nor with recurrent episodes, one-third report recurrent episodes with full remission in between. For the remaining one-third or less, a chronic course with a fluctuating and unremitting course can be expected. Results from the ICPE Surveys revealed that nearly 75\% of individuals who met diagnostic criteria for lifetime MDE reported recurrent episodes [1]. It is noteworthy though that these estimates depend heavily on type of age cohort considered as well as type of depressive disorder. Even in adolescence, unfavorable prognoses have been 
reported for early onset depression and dysthymia (43\% of depression cases and 33\% of dysthymia cases revealed complete remission). Adolescents and young adults with dysthymia have the least favorable outcome, $29 \%$ of those with a baseline dysthymia developed a major depression and 24\% met full diagnostic criteria for dysthymia again at follow-up [34].

\section{Patterns of comorbidity: is anxiety primary?}

Comorbidity is a fundamental characteristic of all anxiety and depressive disorders, and is not confined to some disorders such as GAD, believed to have exceedingly high comorbidity rates. Using data from the cross-sectional NCS, Kessler analyzed subjects with anxiety and depressive disorders regarding 12-month and lifetime comorbidity [20].

As can be seen in Table 1, only up to one-fifth of respondents with a 12-month anxiety disorder did not meet 12- month and lifetime criteria for any other mental disorder surveyed. Therefore, they can be regarded as having a pure anxiety disorder over their lifetime. About the same number of people had a pure anxiety disorder in the 12-month period, yet they had also experienced at least one comorbid disorder prior in their lifetime. The largest proportion of the persons with a 12-month anxiety diagnosis had another disorder in the same 12-month period and lifetime. A similar picture emerged for the affective disorders (Table 2). More than two thirds of subjects with any affective disorders met criteria for at least one other mental disorder in their lifetime and in the 12-month period prior to the investigation. This finding has been replicated in someway or the other by numerous studies [2,24,32,36] and has highlighted not only that anxiety and depressive disorders are closely associated, but also that pure anxiety disorders are quite rare both cross-sectionally and lifetime. Due to the retrospective nature of these data, however, it remained unresolved whether comorbid patterns differ by type of anxiety disorder and patterns of temporal sequence.

Cross-sectional studies of this type are limited to the use of retrospectively reported age of onset to reveal that most anxiety disorders start early and that depressive disorders usually start later in life [32,36]. Recent analyses of the NCS data revealed that more than $80 \%$ of any anxiety disorders are the primary disorder, leaving less than $20 \%$ that occur secondary to another primary disorder [20]. Specific phobias are most frequently primary disorders (68\%) followed by social phobia (63\%), PTSD (52\%), agoraphobia (45\%), GAD (37\%), and panic disorder (23\%). In contrast, depressive disorders less frequently occur as primary disorders. In addition, other studies have consistently shown that major depression emerges after the onset of the anxiety disorder in the majority of cases and, therefore, can be regarded as secondary $[12,32,36]$.

Yet, cross-sectional studies rely on retrospective information of the ages of onset in order to determine the sequence of disorders and, therefore, are handicapped by possible recall biases. Prospective-longitudinal studies are more adequate to ensure the temporal-relationship between anxiety disorders and depression. One of these rare studies is the early developmental stages of psychopathology (EDSP) study carried out in Munich, Germany. This study included one baseline and two follow-up assessments over a period of 5 years.

Fig. 1 gives the proportions of pure anxiety cases and those comorbid with depression for the cohort of 14-17 years old at the baseline investigation. Consistent with relatively low prevalence rates for depression, less than $20 \%$ of cases with an anxiety disorder had ever experienced a comorbid major depression or other threshold depressive disorder. Approximately, 2 years later at the first follow-up, these comorbidity rates almost doubled (not shown), and approximately 5 years later (Fig. 2), at the final follow-up of the EDSP, 
there is a dramatic increase in subjects with an anxiety disorder also meeting criteria for major depression or other threshold depressive disorders. In the majority of cases with either form of specific anxiety disorders, the proportion of comorbid depression in anxiety is clearly over $50 \%$. The highest comorbidity rates with depression can be seen for GAD, separation anxiety disorder (SAD) and panic disorder, yet even the comorbidity rates for specific phobias of any type are only marginally lower. Similar results were recently reported for the entire sample of the EDSP study (14-24 years at baseline; [53]).

Clearly these prospective-longitudinal data in a representative community sample provide much stronger evidence for the predominant pattern of temporal precedence of anxiety disorders over depressive disorders. Despite the fact that this finding fulfills one core requirement in the process of establishing the status of anxiety disorders as a causal risk factor [26], the question of whether this can be interpreted as anxiety disorders being (causal) risk factors for secondary depression remains.

\section{Is anxiety a causal factor and a target for early intervention/prevention of depression?}

What are the potential etiological, pathogenetic, and clinical consequences of the high comorbidity between anxiety disorders and depression? Why do anxious subjects become depressed? Are anxiety disorders risk factors for subsequent depression?

Research regarding risk factors has been hampered by the use of inconsistent terminology such as risk, risk factor and cause, leading to the application of inadequate methods and deceptive interpretations. Kraemer et al. [26] made an attempt to clarify these terms in developing a typology. Within this typology, a correlate is associated with the outcome but does not necessarily precede the outcome. Only if precedence can be proved, the term risk factor may be used.A risk factor, which can be shown to change spontaneously within a subject (e.g. age or weight) or by intervention (e.g. drugs or psychotherapy), can be called variable risk factor. If a change is not possible (e.g. gender, birth cohort, or race), the term fixed marker is proposed to be used. The term causal risk factor is only appropriate, if a variable risk factor can be shown to be manipulable and to change the risk of the outcome. If this is not the case (e.g. genes), the term variable marker should be used. Kraemer et al. also stress that prospective-longitudinal studies are necessary to identify risk factors in order to insure the temporal precedence of the factor and the outcome.

Applying this model to the prospective-longitudinal EDSP data reveals that when using cumulative data over 5 years (Fig. 3), the likelihood of developing a major depression if one has a primary anxiety disorder increases significantly. In the total sample of subjects aged 1424 years at baseline, and up to the age of 29 years, subjects with no anxiety disorder have a relatively low chance of developing a major depression. However, if one has a primary anxiety disorder, or especially a GAD, the risk for secondary depression increases dramatically.

Also, strictly prospective analyses reveal that any pure and primary anxiety disorder as well as the specific anxiety disorders (social phobia, specific phobia, agoraphobia and GAD) at baseline significantly increase the risk of first onset of depression during the 5 year follow-up period [4]. Although GAD appeared to have the strongest association with subsequent depression (Odds ratio (OR) $=4.5$; 95\%- KI $=1.9-10.3$ ), the ORs for the other anxiety disorders are also quite high and consistent. This finding holds true even when adjusting for age, gender, and comorbidity. 
These results are consistent with previous findings from prospective data of the ECA study [15] in adults and older people. The occurrence of specific phobia, agoraphobia, obsessivecompulsive disorder, and panic attacks significantly increased the risk for first onset of major depression, even after adjusting for sociodemographic differences and other psychiatric comorbidity. Goodwin suggested that each anxiety disorder and panic attacks appear to make an independent contribution to the risk of depression.

Our data go beyond these earlier findings, because they were collected prospectively in adolescents and young adults - the key risk period for first onset of anxiety disorders and secondary depression.

However, the pathways leading from anxiety to depression are still not identified. At least two different perspectives have been suggested (see [53]). Frst, anxiety and depression may share identical or at least similar vulnerability and risk factors. According to the "lumpers"perspective, anxiety is merely regarded as a prodromal stage or alternatively as a severity marker of depression. Lumpers usually come to their conclusion using dimensional and crosssectional data $[27,28,50]$. In contrast, the "splitters"-perspective assumes that anxiety and depressive disorders are separate disorders with sufficiently specific and different vulnerability and risk factors. Within this perspective, anxiety disorders are not only important risk factors for the development of depressive disorders, but also this distinction bears important and promising preventive and therapeutic consequences that are believed to be different for each of the anxiety disorders on one hand, and for depression, on the other. As mediating variables for this risk constellation, the splitters assume processes like demoralization, the accumulation of depression-specific risk factors (e.g. avoidance behavior), or an increasing sensitization of cognitive-behavioral and neurobiologic processes.

It is noteworthy that among the anxiety disorders, there is a still considerable controversy regarding the nosological status of GAD and whether GAD should be considered in the same way as other anxiety disorders. The repeated observation that GAD is highly associated with depression has led to the conclusion that both disorders are part of the same spectrum, and that GAD is merely a prodrome, residual, or severity marker of depressive disorders (e.g. $[6,31])$. Some support for this model comes from the similarity of age of onset patterns of GAD and depression, and factor analyses have revealed that GAD appears to be more closely related to depression, than to the other anxiety disorders [27,28,43]. Moreover, twin studies in females have shown that contrary to other anxiety disorders, GAD and depression appear to share a similar set of non-environmental factors, and thus might have a similar genetic disposition [17-19]. However, there is more evidence from recent studies that GAD should be conceptualized as an independent disorder, based on the following observations: GAD and major depressive disorder have different prevalence rates, different patterns of diagnostic overlap, different risk factors, and associated features. Also, substantial impairment has been found for pure GAD not comorbid with depression [8,21,22,52,59].

Recent analyses (a) deal with the underlying mechanisms under which secondary depressions are most likely to develop and (b) examine whether the severity and type of symptoms of secondary depression are different from those of other forms of MDE. Wittchen et al. [53] have analyzed data from a prospective-longitudinal community study and found that the following characteristics of anxiety disorders (at baseline) were significantly associated with an increased risk of secondary depression: the number of anxiety disorders, the impairment, the frequency of avoidance behavior and the presence of panic attacks. Recent findings further suggest that among various clinical characteristics of anxiety disorders (number of comorbid anxiety disorders, impairment, presence of panic attacks) severe impairment 
emerges as the strongest predictor of major depression [4]. Further analyses from the same study have revealed that individuals who have both depressive disorders and social phobia experience a more malignant course of depression compared to respondents with depression or social phobia alone [40]. During the follow-up period, these persons were significantly more likely to experience more intensive suicidal ideation, more depressive symptoms, and a longer duration of MDE. They also had a significantly greater risk of having attempted suicide (see Table 3).

To sum, beyond the finding that anxiety disorders usually precede depression, increasing the risk for incident depression about threefold, even after 4-5 years, the increasingly sharper picture of the various mediating factors and processes make it appear extremely likely that anxiety disorders can be powerful causal risk factors for secondary depression.

\section{Clinical and psychosocial consequences of anxiety-depression comorbidity}

Anxiety disorders and depression are associated with a wide range of psychosocial impairments and disabilities that may differentially affect development processes, such as career, work productivity, partner and interactional integration, and quality of life as well critical health outcome, such as suicidal behavior and treatment. Further, it should be noted that the profile of disabilities may, but must not inevitably, be more pronounced among those affected by both conditions. For instance, in the German health study (GHS) 10.9\% of those with pure GAD and $7.9 \%$ of those with pure major depression reported a reduction of at least $50 \%$ in their activities in the past month [48].Among those with comorbid GAD and major depression, this proportion is substantially higher (23.2\%). Moreover, 34.3\% of pure GAD cases and 20.8\% of the pure subjects with major depression had at least 6 days that were impaired in the past month. But again, respondents with comorbid GAD and major depression were more frequently impaired (47.6\%). Wunderlich et al. [60] investigated the risk for suicide attempts among anxiety and depressive disorders as well as among subjects with comorbid presentations (EDSP). Seventy-nine percent of all suicide attempters had at least two mental disorders. The risk for suicide attempts was significantly increased in subjects with two diagnoses (OR $=3.5$; 95\%-CI: 1.3-9.1), three diagnoses (OR $=6.4$; 95\%-CI: 2.416.9) and more than three diagnoses (OR = 18.4; 95\%-CI: 7.8-43.6). Regarding separate diagnoses, all anxiety (GAD, social phobia, panic attack, agoraphobia, simple phobia, obsessive-compulsive disorder, PTSD) and depressive disorders (MDE, dysthymia) were significantly associated with suicide attempts ( $\mathrm{OR}=2.2$ and higher). Using multivariate analysis of various comorbidity patterns, the authors also found that all significant findings involved comorbidity between anxiety and depressive disorders.

Additionally, anxiety disorders influence the form, severity, and duration of secondary depression (see above; [40]).

\section{Summary and conclusion}

At least when relying on the integrity of epidemiological data and prospective-longitudinal data from studies in the first three decades of life, there is increasing evidence that anxiety disorders are usually primary to the onset of depression, and increase differentially by type, and number of anxiety disorders, the risk for subsequent depression. Anxiety disorders also affect the course of depression in increasing the risk for an earlier onset of depression, which is believed to be more frequently associated with a particularly malignant course, which includes the risk for more intensive suicidal ideation and attempts, and longer duration of depressive episodes. In addition, the number of depressive symptoms are increased in subjects 
with primary anxiety disorders. Further, it can be assumed that the effect size follows doseresponse relationships in showing that a higher number of anxiety disorders, and the associated higher level of severe impairment, additionally elevate the risk for secondary depression. Finally, little doubt remains regarding the question of whether primary anxiety disorders associated with secondary depression increase the risk for a wide range of more severe psychosocial impairments and disabilities.

Given this strong link, it is curious that similar data have only rarely been reported in previous clinical samples. At least four factors seem to contribute to this past omission: first, epidemiological findings, especially in primary care [52], have fairly consistently shown that anxiety disorders are frequently regarded as non-severe disorders, thus they remain much more frequently undiagnosed and untreated in the health care system, as does depression, for example. Anxiety disorders are primarily recognized and treated if they present with comorbid depression. Second, clinical samples in treatment are usually much older than cases identified and studied in epidemiological research, and further most usually present with both conditions, and as such, making a diagnostic separation of the two groups is a clinical challenge, especially due to reliance on retrospective assessments. Third, most clinicians are still not following strictly the DSM-IV and ICD-10 differential diagnostic classification, preferring instead a syndromatic approach that lumps together anxiety and depression. And fourth, clinical randomized studies usually employ a long list of exclusion criteria, thus, a considerable proportion of the most frequent comorbidity patterns are systematically excluded in clinical trials.

\subsection{Implications for further research}

The strong evidence for the case of primary anxiety disorders serving as causal risk constellations has a number of exciting perspectives for both effective prevention and improving early treatment approaches. Based on available evidence, it appears time for major prevention trials. For example, (a) can we change the risk for secondary depression by earlier recognition and treatment of anxiety disorders in the first two or three decades of life? (b) How many incident cases of depression can we avoid by such strategy? (c) Can we change the length of depressive episodes and the risk for recurrence more effectively by targeted intervention on primary and presumably persistent anxiety disorders?

Yet, there remains an unfortunate number of open questions that currently hamper our ability to design such interventions appropriately. Examples include the following:

(1) To date, anxiety disorders could be shown to be variable risk factors according to the strict typology of Kraemer et al. [26]. Admittedly, it remains an unresolved issue whether and why anxiety disorders are causal risk factors for first onset of major depression. Although recent findings from prospective epidemiological studies suggest a causal role of anxiety disorders in the etiology of depressive disorders, the mediating processes remain largely unknown. Future targeted clinical and epidemiological studies are needed to clearly answer these questions.

(2) Are all anxiety disorders the same? Findings suggest that there are differences in the temporal-relationships between the specific anxiety disorders and depression. For example, specific phobia is consistently shown to have its onset primary to depression in about $80 \%$ of cases whereas GAD only occurs prior to depression in about 35\% of cases. Despite this finding, all anxiety disorders increase the risk of subsequent depression significantly whereas GAD seems to have the strongest association. 
(3) To what degree do specific anxiety disorders and depression have different or shared vulnerability and risk factors? Some findings favor the perspective that there is at least significant evidence for the separation of various anxiety disorders from major depression in terms of risk factors and natural course. Nevertheless, this problem remains unresolved, since there are also contrary findings (e.g. findings from twin studies).

\subsection{Public health implications}

As findings consistently show, the prevalence of depressive disorders is high, even in young people [29,58], and there are enormous associated economic health costs and impairments [37]. Thus, effective strategies for prevention are increasingly important. Prevention trials are rare, and mainly characterized by unspecific intervention modules that do not target specific vulnerability and risk factors of depressive disorders. Most studies have found only moderate effect sizes $[9,13,35]$. Nevertheless, keeping in mind that primary anxiety disorders are risk factors for secondary depression, and that the presence of anxiety disorders has considerably adverse effects on the course of depression, some implications for prevention and clinical management can be outlined. In particular, early intervention programs may be promising. Specifically, it should be investigated whether the treatment of early primary anxiety disorders can prevent onset of secondary depression and other complications and impairments. Approximately, $10 \%$ of mood disorders may be prevented by successful early intervention with social phobia [25]. Explorative analyses of the EDSP data have revealed that if all anxiety disorders among 14-24 years old would be successfully treated one would be able to prevent $43 \%$ of all depressive episodes in early adulthood (attributable population risk fraction, lower limit). Preliminary results are promising in showing that treatment of panic attacks may reduce the risk of developing major depression [14]. Randomized clinical studies with a longitudinal design over at least 3 years are needed next to explore whether treatment of anxiety disorders decreases the risk of subsequent major depression. Within routine clinical care, it is necessary to always examine prior histories of anxiety disorders in depression and to consider unfavorable effects of undetected anxiety for the course of depression. Therefore, a more comprehensive life-cycle assessment of mental disorders appears to be needed to improve treatment and management strategies.

Table 1

Pure and Comorbid Anxiety Disorders

\begin{tabular}{lcccc}
\hline & & \multicolumn{2}{c}{ Proportion among 12-mo disorders (\%) } \\
& 12-mo prevalence & Pure in 12-mo \& lifetime & Pure in 12-mo \& comorbid in lifetime & Comorbid in 12-mo \& lifetime \\
\hline Anxiety disorder & 3.2 & 7.8 & 9.6 & 82.6 \\
GAD & 2.3 & 3.8 & 8.1 & 88.1 \\
Panic disorder & 7.8 & 17.4 & 14.4 & 68.1 \\
Social phobia & 8.8 & 15.9 & 15.9 & 68.3 \\
Specific phobia & 3.9 & 9.4 & 8.4 & 82.2 \\
Agoraphobia & 3.9 & 17.8 & 12.6 & 69.5 \\
PTSD & 19.3 & 21.5 & 19.9 & 58.6 \\
Any Anxiety & & & \\
\hline
\end{tabular}


Table 2

Pure and Comorbid Affective Disorders

\begin{tabular}{lcccc}
\hline & \multicolumn{2}{l}{ Proportion among 12-mo disorders (\%) } \\
& 12-mo prevalence & Pure in 12-mo \& lifetime & Pure in 12-mo \& comorbid in lifetime & Comorbid in 12-mo \& lifetime \\
\hline Mood disorders & 10.2 & 15.6 & 14.4 & 69.9 \\
Major depressive episode & 2.7 & 9.6 & 2.4 & 88.0 \\
Dysthymia & 1.4 & 0.0 & 1.9 & 98.1 \\
Bipolar I disorder & 11.4 & 16.3 & 13.8 & 69.9 \\
Any mood disonder & & &
\end{tabular}

Adapted from: Kessler (1997).

Tatle 3

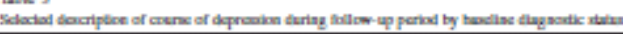

\begin{tabular}{|c|c|c|c|c|c|c|c|c|c|c|c|c|c|c|c|c|}
\hline \multirow[t]{2}{*}{ Hancline daysoutide thas } & \multicolumn{3}{|c|}{ Salcidallity ${ }^{2}$} & \multicolumn{4}{|c|}{ 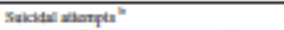 } & \multicolumn{3}{|c|}{ 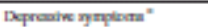 } & \multicolumn{3}{|c|}{ Depronins quanda } & \multicolumn{3}{|c|}{ 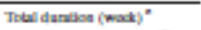 } \\
\hline & Mas & $(\tan x)$ & pastc미 & Namba & $(\mathbf{x})$ & Da & $\max -\operatorname{con}$ & Man & $(I R R)^{2}$ & [PELCD] & Mean & (IRR) & [Desect] & $\mathrm{Man}$ & $(5 x)$ & $\operatorname{ses}-$ - \\
\hline 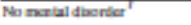 & 0.94 & & & 4 & $(4.17)$ & 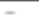 & & 10.20 & & & 2019 & & & $5 \pi$ & & \\
\hline 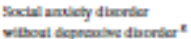 & $0.0 \mathrm{x}$ & (L.1) & $p 6-1.9$ & 3 & $(2.29)$ & 26 & $10,-159$ & 12.14 & (1.2) & {$[1.0-1.5]$} & 447 & (10) & {$[0+24]$} & 66.19 & (1.) & $0.7-409$ \\
\hline 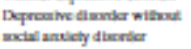 & $0 . \%$ & (1.0) & $0.7-1.91$ & 3 & (457) & 1.1 & pas $-4 n$ & 12.85 & (12) & [1.2-1.4] & $20 x$ & (ه) & {$[03-1,4]$} & $n n$ & (1.1) & 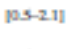 \\
\hline 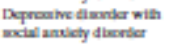 & $1 . \mathrm{s}$ & (22) & {$[1.4-14]$} & 6 & $(12.70)$ & 70 & $n 12-3210$ & 14.95 & (1.4) & [1.2-1.7] & 443 & (1.7) & {$[0,-2.4]$} & 5.75 & (a2) & $10-690$ \\
\hline Dpromine alandar with & & (2.1) & $n=3-3 \mid$ & - & & a. & {$[12-\pi 2.21$} & & (1.2) & {$[1.0-1.4]$} & & (29) & {$[0.5-2.2]$} & & (23) & 10.369 \\
\hline
\end{tabular}

Deprome alanda wih

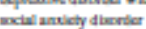

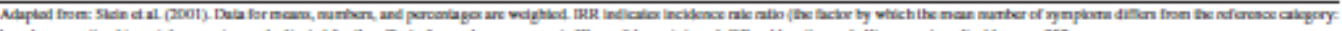

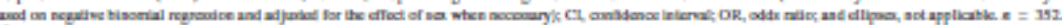

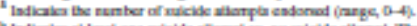

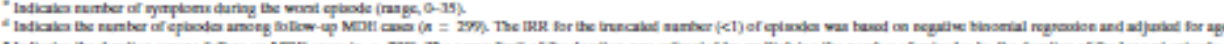

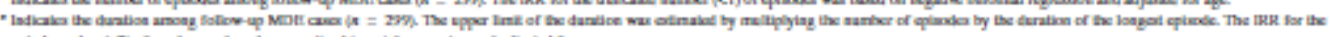

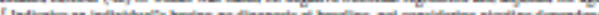

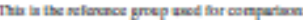

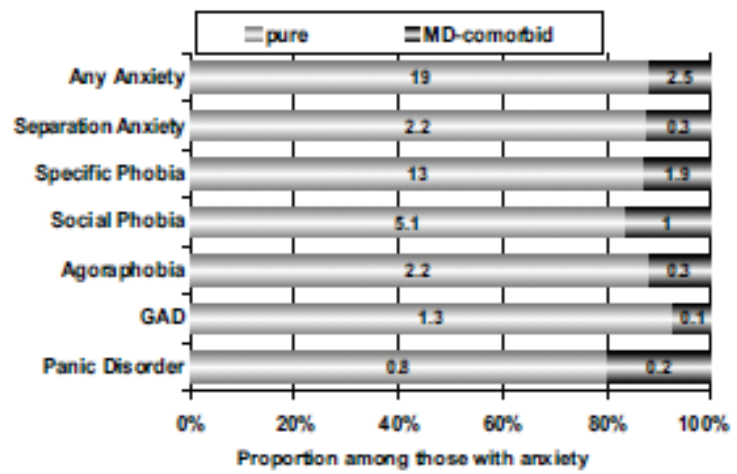

Fig. 1. Proportions of pure and depression-comorbid anxiety disorders among 14-17 years old: baseline.

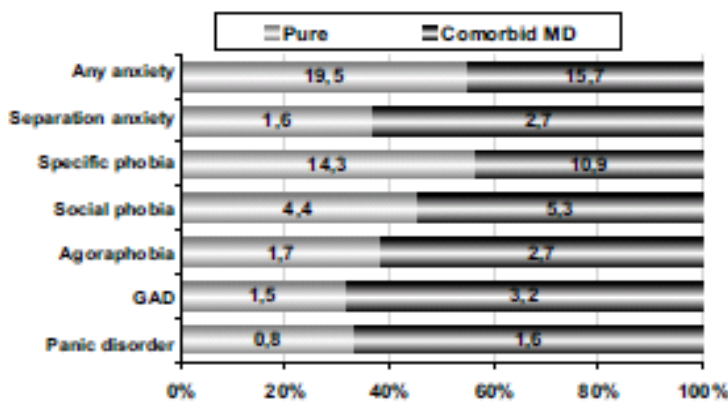

Fig. 2. Five years later - how many anxiety disorders are still pure and how many comortid? 


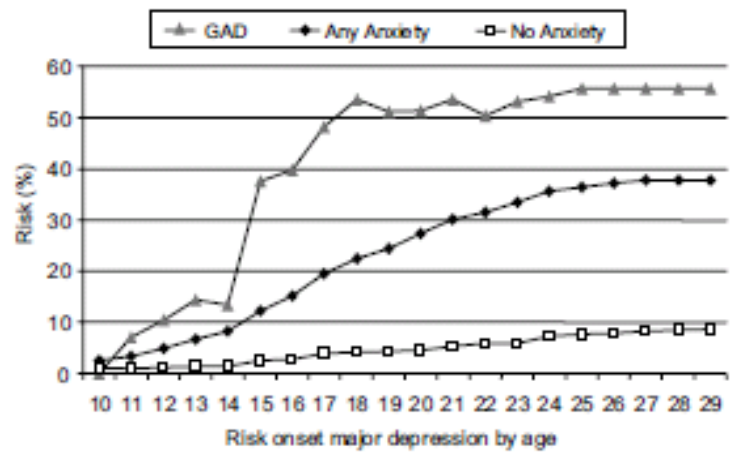

Fig. 3. How likely is first onset of depression if you have a primary anxiety disorder? Cumulative risk over 5 years by age.

\section{References}

[1] Andrade L, Caraveo-Anduaga JJ, Berglund P, Bijl R, de Graaf R, Vollebergh WAM, et al. The epidemiology of major depressive episodes: results from the International Consortium of Psychiatric Epidemiology (ICPE) Surveys. Int J Meth Psychiatr Res 2003;12:3-21.

[2] Angst J. Comorbidity of mood disorders: a longitudinal prospective study. Br J Psychiatry 1996;168:31-7.

[3] Bijl RV, Ravelli A,Van Zessen G. Prevalence of psychiatric disorder in the general population: results of the Netherlands Mental Health Survey and Incidence Study (NEMESIS). Soc Psychiatry Psychiatr Epidemiol 1998;33:587-95.

[4] Bittner A, Goodwin RD, Wittchen H-U, Beesdo K, Höfler M, Lieb R. What characteristics of primary anxiety disorders predict subsequent major depression? J Clin Psychiatry, submitted for publication.

[5] Blazer DG, Hughes D, George LK, Swartz M, Boyer R. Generalized anxiety disorder. In: Robins LN, Regier DA, editors. Psychiatric disorders in America: the epidemiologic catchment area study. New York (NY): The Free Press; 1991. p. 180-203.

[6] Breier A, Charney DS, Heninger GR. The diagnostic validity of anxiety disorders and their relationship to depressive illness. Am J Psychiatry 1985;142:787-97.

[7] Burke KC, Burke JD, Regier DA, Rae DS. Age at onset of selected mental disorders in five community populations. Arch Gen Psychiatry 1990;47:511-8.

[8] Carter RM, Wittchen H-U, Pfister H, Kessler RC. One-year prevalence of subthreshold and threshold DSM-IV generalized anxiety disorder in a nationally representative sample. Depress Anxiety 2001; 13:78-88.

[9] Clarke GN, Hornbrook M, Lynch F, Polen M, Gale J, Beardslee WR, et al. A randomized trial of a group cognitive intervention for preventing depression in adolescent offspring of depressed parents. Arch Gen Psychiatry 2001;58:1127-34.

[10] Eaton WW, Dryman A, Weissman MM. Panic and phobia. In: Robins LN, Regier DA, editors. Psychiatric disorders in America The epidemiologic catchment area study. NewYork (NY): The Free Press; 1991. p. 155-79.

[11] Essau CA, Karpinski NA, Petermann F, Conradt J. Häufigkeit und Komorbidität psychischer Störungen bei Jugendlichen: Ergebnisse der Bremer Jugendstudie. Z Klin Psychol Psychother 1998;46:105-24.

[12] Essau CA, Karpinski NA, Petermann F, Häufigkeit Conradt J. Komorbidität und psychosoziale Beeinträchtigung von Depressiven Störungen bei Jugendlichen: Ergebnisse der Bremer Jugendstudie. Z Klin Psychol Psychother 1998;46:316-29.

[13] Gillham JG, Reivich KJ. Prevention of depressive symptoms in school children. A research update: two-year follow-up. Psychol Sci 1999;6:343-51.

[14] Goodwin R, Olfson M. Treatment of panic attack and risk of major depressive disorder in the community. Am J Psychiatry 2001;158:1146-8.

[15] Goodwin RD. Anxiety disorders and the onset of depression among adults in the community. Psychol Med 2002;32:1121-4. 
[16] Jacobi F,Wittchen H-U, Hölting C, Höfler M, Pfister H, Müller N, et al. Prevalence, comorbidity and correlates of mental disorders in the general population: results from the German Health Interview and Examination Survey (GHS). Psychol Med (in press).

[17] Kendler KS. Major depression and generalised anxiety disorder. Same genes, (partly) different environments-revisited. Br J Psychiatry 1996; 168:68-75.

[18] Kendler KS, Neale MC, Kessler RC, Heath AC, Eaves LJ. Major depression and generalized anxiety disorder. Same genes, (partly) different environments? Arch Gen Psychiatry 1992;49:716-22. [19] Kendler KS, Walters EE, Neale MC, Kessler RC, Heath AC, Eaves LJ. The structure of the genetic and environmental risk factors for six major psychiatric disorders in women. Arch Gen Psychiatry 1995;52: 374-83.

[20] Kessler RC. The prevalence of psychiatric comorbidity. In: Wetzler S, Sanderson WC, editors. Treatment strategies for patients with psychiatric comorbidity. NewYork: Wiley; 1997. p. 23-48. [21] Kessler RC, Berglund PA, DeWit DJ, Üstün TB, Wang PS, Wittchen H-U. Distinguishing generalized anxiety disorder from major depression: prevalence and impairment from current pure and comorbid disorders in the US and Ontario. Int J Meth Psychiatr Res 2002; 11:99-111.

[22] Kessler RC, DuPont RL, Berglund P, Wittchen H-U. Impairment in pure and comorbid generalized anxiety disorder and major depression at 12 months in two national surveys. Am J Psychiatry 1999;156:1915-23.

[23] Kessler RC, McGonagle KA, Zhao S, Nelson CB, Hughes M, Eshleman S, et al. Lifetime and 12month prevalence of DSM-III-R psychiatric disorders in the United States: results from the National Comorbidity Survey. Arch Gen Psychiatry 1994;51:8-19.

[24] Kessler RC, Nelson CB, McGonagle KA, Liu J, Swartz M, Blazer DG. Comorbidity of DSM-III$\mathrm{R}$ major depressive disorder in the general population: results from the US National Comorbidity Survey. Br J Psychiatry 1996;168:17-30.

[25] Kessler RC, Stang P, Wittchen H-U, Stein MB, Walters EE. Lifetime comorbidities between social phobia and mood disorders in the US National Comorbidity Survey. Psychol Med 1999;29:55567.

[26] Kraemer HC, Kazdin AE, Offord DR, Kessler RC, Jensen PS, Kupfer DJ. Coming to terms with the terms of risk. Arch Gen Psychiatry 1997;54:337-43.

[27] Krueger RF. The structure of common mental disorders. Arch Gen Psychiatry 1999;56:921-6.

[28] Krueger RF, Caspi A, Moffitt TE, Silva PA. The structure and stability of common mental disorders (DSM-III-R): a longitudinal-epidemiological study. J Abnorm Psychol 1998;107:216-27. [29] Lewinsohn PM, Hops H, Roberts RE, Seeley JR, Andrews JA. Adolescents psychopathology: I. Prevalence and incidence of depression and other DSM-III-R disorders in high school students. J Abnorm Psychol 1993;102:133-44.

[30] Magee WJ, Eaton WW, Wittchen H-U, McGonagle K, Kessler RC. Agoraphobia, simple phobia, and social phobia in the National Comorbidity Survey. Arch Gen Psychiatry 1996;53:159-68.

[31] Massion AO, Warshaw MG, Keller MB. Quality of life and psychiatric morbidity in panic disorder and generalized anxiety disorder. Am J Psychiatry 1993;150:600-7.

[32] Merikangas KR, Angst J, Eaton W, Canino G, Rubio-Stipec M, Wacker H, et al. Comorbidity and boundaries of affective disorders with anxiety disorders and substance misuse: results of an international task force. Br J Psychiatry 1996;168:58-67.

[33] Meyer C, Rumpf H-J, Hapke U, Dilling H, John U. Lebenszeitprävalenz psychischer Störungen in der erwachsenen Allgemeinbevölerung. Ergebnisse der TOCAS-Studie. Nervenarzt 2000;71:53542.

[34] Oldehinkel AJ, Wittchen H-U, Schuster P. Prevalence, 20-month incidence and outcome of unipolar depressive disorders in a community sample of adolescents. Psychol Med 1999;29:655-68. [35] Pattison C, Lynd-Stevenson RM. The prevention of depressive symptoms in children: the immediate and long-term outcomes of a schoolbased program. Behav Change 2001;18:78-102. [36] Regier DA, Rae DS, Narrow WE, Kaelber CT, Schatzberg AF. Prevalence of anxiety disorders and their comorbidity with mood and addictive disorders. Br J Psychiatry 1998;173:24-8.

[37] Rice DP, Miller LS. Health economics and cost implications of anxiety and other mental disorders in the United States. Br J Psychiatry 1998;173:4-9.

[38] Robins LN, Regier DA. Psychiatric disorders in Amerika: the Epidemiologic Catchment Area Study. New York (NY): The Free Press; 1991. 
[39] Schuster P. Depressionen bei Jugendlichen und jungen Erwachsenen. Eine klinischepidemiologische Analyse. Stuttgart: Schattauer Verlag; 2001 Mit einem Geleitwort von Prof. Dr. Dipl.-Psych. Hans-Ulrich Wittchen.

[40] Stein MB, Fuetsch M, Müller N, Höfler M, Lieb R, Wittchen H-U. Social anxiety disorder and the risk of depression. A prospective community study of adolescents and young adults. Arch Gen Psychiatry 2001;58:251-6.

[41] Turner RJ, Gil AG. Psychiatric and substance use disorders in south Florida: racial/ethnic and gender contrasts in a young adult cohort. Arch Gen Psychiatry 2002;59:43-50.

[42] Vega WA, Kolody B, Aguilar-Gaxiola S, Alderete E, Catalano R, Caraveo-Anduaga J. Lifetime prevalence of DSM-III-R psychiatric disorders among urban and rural mexican americans in California. Arch Gen Psychiatry 1998;55:771-8.

[43] VolleberghWAM, Iedema J, Bijl RV, de Graaf R, Smit F, Ormel J. The structure and stability of common mental disorders. Arch Gen Psychiatry 2001;58:597-603.

[44] Weissman MM, Bland RC, Canino GJ, Faravelli C, Greenwald S, Hwu H-G, et al. The crossnational epidemiology of panic disorder. Arch Gen Psychiatry 1997;54:305-9.

[45] Wittchen H-U. Der Langzeitverlauf unbehandelter Angststörungen: Wie häufig sind Spontanremissionen? Verhaltensther 1991;1:273-82.

[46] Wittchen H-U. What is comorbidity—fact or artefact? Br J Psychiatry 1996;168:7-8.

[47] Wittchen H-U. The many faces of social anxiety disorder. Int Clin Psychopharmacol 2000;15:712.

[48] Wittchen H-U, Carter RM, Pfister H, Montgomery SA, Kessler RC. Disabilities and quality of life in pure and comorbid generalized anxiety disorder and major depression in a national survey. Int Clin Psychopharmacol 2000;15:319-28.

[49] Wittchen H-U, Fehm L. Epidemiology, patterns of comorbidity, and associated disabilities of social phobia. Psychiatr Clin North Am 2001;24:617-41.

[50] Wittchen H-U, Höfler M, Merikangas KR. Toward the identification of core psychopathological processes? Arch Gen Psychiatry 1999;56: 929-31.

[51] Wittchen H-U, Hoyer J. Generalized anxiety disorder: nature and course. J Clin Psychiatry 2001;62:15-9.

[52] Wittchen H-U, Kessler RC, Beesdo K, Krause P, Höfler M, Hoyer J. Generalized anxiety and depression in primary care: prevalence, recognition and management. J Clin Psychiatry 2002;63:2434.

[53] Wittchen H-U, Kessler RC, Pfister H, Lieb R. Why do people with anxiety disorders become depressed? A prospective-longitudinal community study. Acta Psychiatr Scand 2000;102:14-23. [54] Wittchen H-U, Lecrubier Y, Beesdo K, Nocon A. Relationships among anxiety disorders: patterns and implications. In: Nutt DJ, Ballenger JC, editors. Anxiety disorders. Oxford: Blackwell Science; 2003. p. 25-37.

[55] Wittchen H-U, Lieb R, Pfister H, Schuster P. The waxing and waning of mental disorders: evaluating the stability of syndromes of mental disorders in the population. Compr Psychiatry 2000;41:122-32.

[56] Wittchen H-U, Lieb R, Schuster P, Oldehinkel AJ. When is onset? Investigations into early developmental stages of anxiety and depressive disorders. In: Rapoport JL, editor. Childhood onset of "adult” psychopathology, clinical and research advances.Washington: American Psychiatric Press; 1999. p. 259-302.

[57] Wittchen H-U, Mühlig S, Pezawas L. Natural course and burden of bipolar disorders. Int J Neuropharmacol 2003;6:145-54.

[58] Wittchen H-U, Nelson CB, Lachner G. Prevalence of mental disorders and psychosocial impairments in adolescents and young adults. Psychol Med 1998;28:109-26.

[59] Wittchen H-U, Zhao S, Kessler RC, Eaton WW. DSM-III-R generalized anxiety disorder in the National Comorbidity Survey. Arch Gen Psychiatry 1994;51:355-64.

[60] Wunderlich U, Bronisch T, Wittchen H-U. Comorbidity patterns in adolescents and young adults with suicide attempts. Eur Arch Psychiatry Clin Neurosci 1998;248:87-95. 